\title{
Fast compression of a current sheet during externally driven magnetic reconnection
}

\author{
Y. Ono, M. Inomoto, Y. Ueda, T. Matsuyama, and Y. Murata
}

High Temperature Plasma Center, University of Tokyo, Tokyo 113-8656, Japan

(Received June 20, 2000; Revised January 5, 2001; Accepted February 28, 2001)

\begin{abstract}
Magnetic reconnection of two toroidal plasmas with arbitrary $q$ values revealed a dependence of sheet-current dissipation and ion heating on ion-gyromotion. Effective sheet-current resistivity was found to increase significantly, when an external force compressed the current sheet shorter than the ion-gyroradius, and both the reconnection speed and ion temperature increased with decreasing current sheet width and with increasing ion-gyroradius.
\end{abstract}

\section{Introduction}

Magnetic reconnection has been investigated as important key physics for explaining global restructuring of various magnetized plasmas, such as the solar corona, the magnetotail and various fusion plasmas. Its physics contains not only magnetohydrodynamic (MHD) effects but also several "anomalous" effects which are related to particle and two fluid motions. Recently, the solar satellite "Yohkoh" and the magnetospheric satellite "Geotail" have measured many signatures of anomalous dissipation and heating effects of magnetic reconnection (Shimizu et al., 1994; Saito et al., 1995; Tsuneta, 1996). Speeds of these reconnections are much higher than that of the Sweet-Parker model (Sweet, 1953; Parker, 1957), suggesting that their effective sheet-current resistivities are a few order higher than the classical resistivity. It leads to anomalous release of magnetic energy into plasma kinetic and thermal energies during preferential heating of solar coronas (Shimizu et al., 1994; Tsuneta, 1996). The current sheet in the reconnecting magnetotail lobes has been observed to have their ion temperature higher than electron temperature (Saito et al., 1995). Theoretically, their current sheet widths have been predicted to be on the order of the ion-gyroradius (Truemann and Baumjohann, 1997). It is noted that reconnection of the southward interplanetary magnetic field (IMF) with the northward dipole field of the earth is faster than that of the northward IMF. The latter has a much larger field component $B_{X}$ parallel to the "X-point" line than the former. Also in periodic/continuous reconnection of laboratory plasmas, the plasma resistivity, magnetic fluctuation amplitude, and temperature ratio of ions to electrons are significantly higher in RFPs (Reversed Field Pinches) with small $B_{X}$ than in ohmic heating tokamaks with large $B_{X}$. An important question then arises: by what mechanism does the reconnection speed/heating depend on $B_{X}$ ? Is it related to the ion-gyromotion determined by $B_{X}$ and current-sheet-compression? Mechanisms for the anoma-

Copy right(c) The Society of Geomagnetism and Earth, Planetary and Space Sciences (SGEPSS); The Seismological Society of Japan; The Volcanological Society of Japan; The Geodetic Society of Japan; The Japanese Society for Planetary Sciences. lous dissipation have been investigated by macroparticle simulations (Leboeuf et al., 1982; Tajima and Sakai, 1989; Horiuchi and Sato, 1997; Shay et al., 1998) and their effects on spatial field structures by various MHD simulations (Sato and Hayashi, 1979; Biskamp, 1986; Sato et al., 1992). In RFPs, several experimental groups have measured magnetic fluctuations caused by reconnection and their mode cascade up to the ion-gyro-mode number as a cause for the anomalous ion heating (Scime et al., 1992). Stenzel and Gekelman first demonstrated laboratory reconnection with current sheet widths shorter than the ion-gyroradius (Stenzel et al., 1982; Gekelman and Pfister, 1988). They reported non-MHD/particle effects, such as Whistler wave activities. However, the relation of anomalous resistivity/ion heating to the sheet-compression process and its dependence on the $B_{X}$ component are left unsolved. We have been reporting that the counterhelicity merging of two spheromaks with merging angle $\theta \approx 180^{\circ}$ is faster than the cohelicity merging with $\theta \approx 90^{\circ}$, both in the fast and slow reconnection regimes using the TS-3 (Ono et al., 1993, 1996) and the MRX devices (Yamada et al., 1997), respectively. Recently, our new tokamak/spheromak/RFP merging with wide range of $q$ values was found to be more effective when the $B_{X}$ field component was varied continuously from 0 to $5 B_{\|}$. As shown in Fig. 1, this method enables us to keep constant the reconnecting field component $B_{\|}$. The external force for sheet-current compression is provided by an acceleration-coil current, enabling us to vary the reconnection speed widely. This paper addresses two important issues: (1) how the resistivity of a current sheet is related to its sheet-current compression in terms of external compressing force and $B_{X}$ field component, (2) how the anomalous current sheet dissipation is related to anomalous ion-heating $\left(T_{i}>T_{e}\right)$ of reconnection. High-resolution measurements of the current-sheet and ion temperature revealed a relationship between the effective sheet-current resistivity and the current-sheet width normalized by the ion-gyroradius. 
RFPs, spheromaks or tokamaks

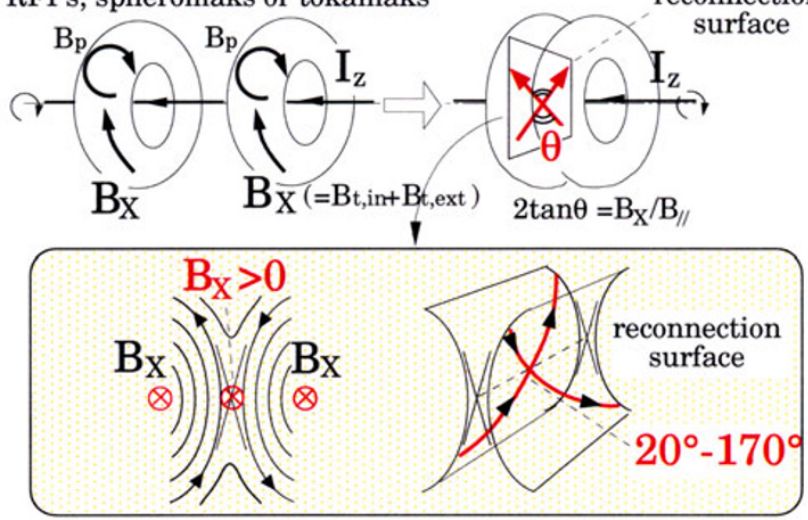

Fig. 1. Control of $B_{X}$ component of magnetic reconnection using two merging tokamak, spheromaks and RFPs (Reversed Field Pinches).

\section{Experimental Setup}

As shown in Fig. 2, the TS-3 merging device (Ono et al., 1993) has two internal poloidal field (PF) coils and two sets each of eight pairs of electrodes to produce poloidal and toroidal fluxes of two separate toroids. A center torus coil with diameter of $12 \mathrm{~cm}$ is located along the center geometric axis to produce the toroidal field $B_{t, e x t}$. Right after formation, the two plasma toroids have major and minor radii of $18 \mathrm{~cm}$ and $12 \mathrm{~cm}$, respectively. The initial ion and electron temperatures $\left(T_{i}\right.$ and $\left.T_{e}\right)$ are about $10 \mathrm{eV}$ and $5 \mathrm{eV}$, respectively. The electron density $n_{e}$ is order of $10^{19} \mathrm{~m}^{-3}$ in the bulk plasma and $0.5-1 \times 10^{20} \mathrm{~m}^{-3}$ inside the current sheet. After the two toroid formation, the reversed currents of PF coils are also used as an acceleration-coil currents $I_{a c c}$ to push the two toroids together. A 2-D array of magnetic probes is

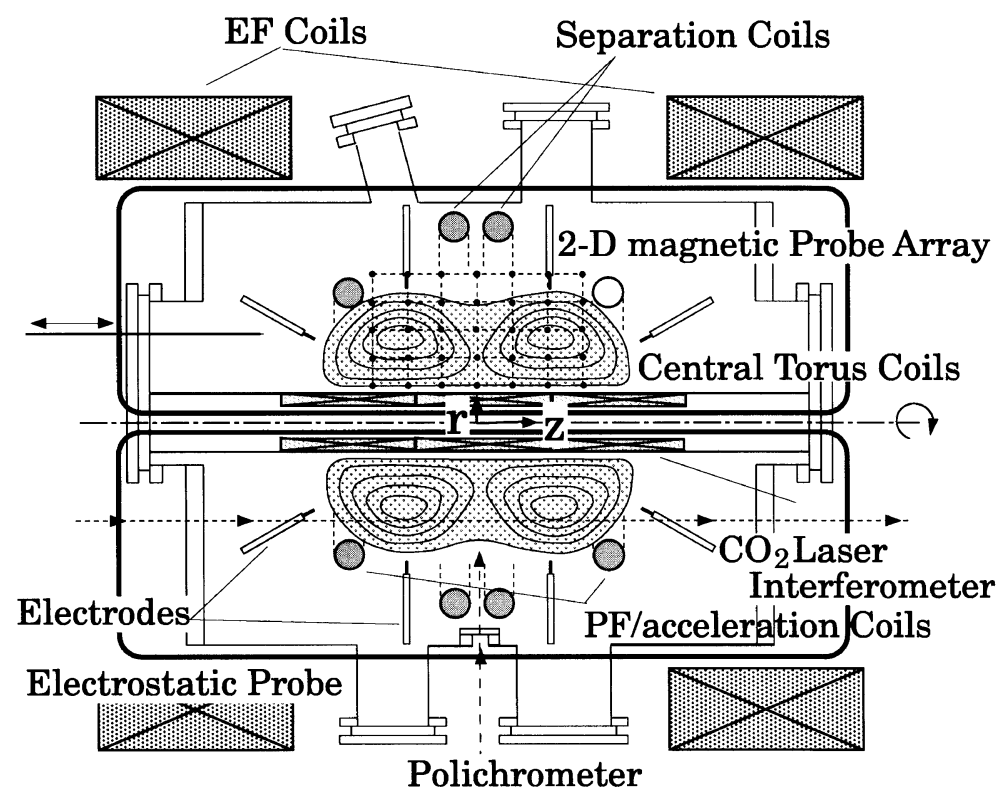

Fig. 2. Experimental setup of TS-3 merging/reconnection device.

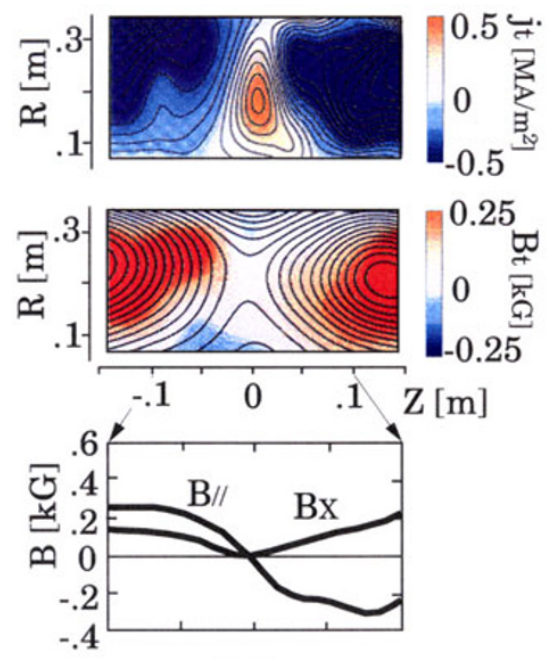

(a)
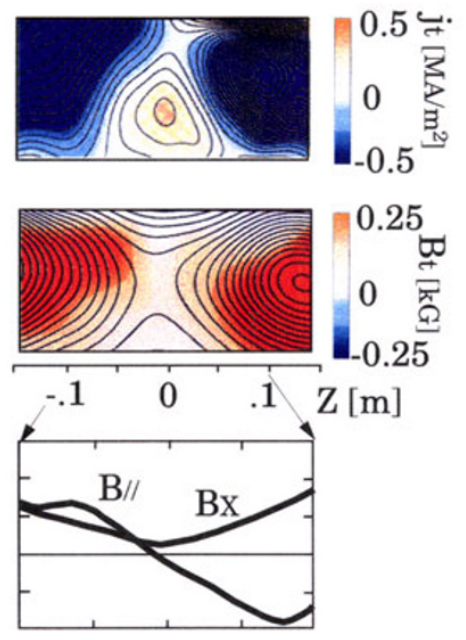

(b)
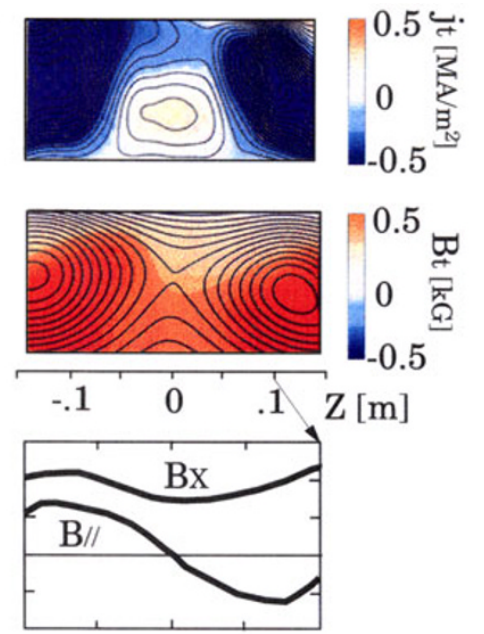

(c)

Fig. 3. 2-D contours of poloidal flux and toroidal field (red and blue colors) of two merging (a) RFPs (Reversed Field Pinches), (b) spheromaks, and (c) tokamaks and their axial profiles of reconnection field $B_{\|}$and field component $B_{X}$ parallel to the X-point line. 
located on an $r-z$ plane of the vessel to measure profiles of the magnetic field $\left(B_{z}, B_{r}, B_{t}\right)$ profiles within $1 \%$ accuracy (typically smaller than $0.5 \%$ ). The accuracy of measured $B$ field is assured by probe calibration/orientation insides the vacuum vessel (after its installation), digitizers with 12 bit resolution and adjustable amplifiers to maximize the dynamic range of digitizers. Based on the 2-D magnetic field profile, 2-D contours of poloidal flux $\Psi$ and toroidal current density $j_{t}$ are calculated on each single discharge, as shown in Ono et al. (1993). The spatial resolution is as fine as $5 \mathrm{~mm}$ in the axial direction and $3 \mathrm{~cm}$ in the radial direction around current sheet. This resolution is fine enough to measure the present current sheet whose typical size is $2 L \approx 15-20 \mathrm{~cm}$ and $2 \delta \approx 3-10 \mathrm{~cm}$, as shown in Fig. 3. Scans of the 2-D probe allowed us to improve probe resolutions further to $2 \mathrm{~mm}$ to check how this major error factor affects the magnetic field measurement. Using this technique, the measurement errors of $j_{t}$ and $\Psi$ are found to be smaller than $10 \%$ and $2 \%$ respectively. A $1 \mathrm{~m}$ polychromater with a $2-\mathrm{D}$ CCD $(584 \times 386)$ detector array is located on the midplane to measure a ion temperature $T_{i}$ profile with wavelength resolution of 0.01 nm (Ono et al., 1993). The $H_{\beta}$ line is used to measure the fast $(10 \mu \mathrm{sec})$ increase in ion temperature during the reconnection because the charge exchange time between $H$ and $H^{+}: 0.1 \mu \mathrm{sec}$ is much shorter than the equi-partition time between the impurity ions and hydrogen ions. Thirty lines of sights perpendicular to the plasma outflow direction are located horizontally on the midplane (Morita et al., 1995; Ono et al., 1996; Murata et al., 2000; Balandin et al., 2001). For each single discharge, the radial $T_{i}$ (parallel to the Xline) profile is calculated by applying the Abel inversion to the measured line-integrated signals from the outer edge to the X-point (or further inside). An electrostatic probe was inserted axially at $r=14 \mathrm{~cm}$ to measure the axial profile of the electron temperature $T_{e}$ and density $n_{e} . \mathrm{A} \mathrm{CO}_{2}$ laser interferometer was used to calibrate the absolute value of $n_{e}$.

\section{Experimental Results}

An external toroidal field $B_{t, \text { ext }}$ is applied to two merging toroids with parallel internal toroidal fields $B_{t, \text { in }}$ to form arbitrary $B_{X}\left(=B_{t, \text { ext }}+B_{t, \text { in }}\right)$ around a toroidal X-point line. The $B_{X}$ component was varied continuously from the RFP regime ( $\left.B_{X} \leq B_{\|}\right)$shown in Fig. 3(a) to the tokamak regime $\left(B_{X} \gg B_{\|}\right)$in Fig. 3(c). Figures 4(a), (b), and (c) show the dependence of reconnection rate $\gamma$ on the field component $B_{X}$, when current sheets of the merging toroids are compressed by the acceleration-coil currents $I_{a c c} \approx 13$ $\mathrm{kA}, 10 \mathrm{kA}$ and $6 \mathrm{kA}$, respectively. The $\gamma$ value is a timeaveraged growth rate of $\alpha$ defined as $\gamma=\frac{d \alpha}{d t} / \alpha$, where $\alpha$ is the ratio of reconnected poloidal flux to total poloidal flux (common flux ratio) (Ono et al., 1993). We kept equal the initial poloidal flux of each merging toroid for this scan, realizing an equal (within $10 \%)$ initial $B_{\|}\left(=B_{\| 0}\right)$ condition. The reconnection rate $\gamma$ is observed to increase with increasing external compressing force provided by $I_{a c c}$. The $\gamma$ value for $I_{a c c}=13 \mathrm{kA}$ is about 2.1 times larger than that for $I_{a c c}=6 \mathrm{kA}$, if $B_{X} / B_{\| 0}=2$. All three curves also agree that $\gamma$ increases inversely with $B_{X}$. In Fig. 4(a), the reconnection with $B_{X} / B_{\| 0}=1$ is about 1.3 times faster than that with $B_{X} / B_{\| 0}=3.5$. It is noted that abrupt changes in $\gamma$ are observed around $B_{X} / B_{\| 0}=2.5$ in (a), 1.8 in (b), and 1 in (c).

This large increase in $\gamma$ is connected with the rise in ion temperature. Figures 5(a), (b) and (c) show the peak ion temperatures $T_{i 0}$ before and after reconnection (at $t=20$ $\mu \mathrm{sec}$ and at $t=35 \mu \mathrm{sec}$, respectively) as a function of $B_{X} / B_{\| 0}$. The ion temperature profile tend to peak around the O-point right after the reconnection. The ion temperature $T_{i 0}$ after reconnection increases with decreasing $B_{X}$ and with increasing $I_{a c c}$. If $B_{X} / B_{\| 0} \approx 2$, the $T_{i}$ increment increases from $20 \mathrm{eV}$ to $105 \mathrm{eV}$, as $I_{a c c}$ is increased from $6 \mathrm{kA}$ to 13 $\mathrm{kA}$. If $I_{a c c} \approx 13 \mathrm{kA}$, it increases from $30 \mathrm{eV}$ to $105 \mathrm{eV}$, as $B_{X} / B_{\| 0}$ is decreased from 3.5 to 1 . The electron temperature stays around 5-10 eV during each reconnection. In the case of $B_{X} / B_{\| 0} \approx 1$ in Fig. 5(a), the ion heating energy is as large as $80 \%$ of the dissipated magnetic energy, suggesting the preferential ion heating effect of reconnection.

An important question is why the reconnection speed as well as the ion heating energy depend on $B_{X} / B_{\| 0}$ and $I_{a c c}$. Figures $6(\mathrm{a}),(\mathrm{b})$ and (c) show time evolutions of common flux ratio $\alpha$, ion temperature $T_{i, X}$ at the X-point, currentsheet width $2 \delta$, and ion-gyroradius $\times 2$ (diameter) $2 \rho_{i}$ at the X-point, and effective resistivity $\eta_{X}$ at the X-point, for three different reconnections: (a) $B_{X} \approx 1.0 B_{\| 0}, I_{a c c} \approx 5$ $\mathrm{kA}$, (b) $B_{X} \approx 1.0 B_{\| 0}, I_{a c c} \approx 10 \mathrm{kA}$, and (c) $B_{X} \approx 2.7 B_{\| 0}$, $I_{a c c} \approx 10 \mathrm{kA}$. The ion-gyroradius $\rho_{i}$ is calculated from the magnetic field $B_{X}$ and ion temperature $T_{i, X}$ measured at the $\mathrm{X}$-point. After confirming null velocity $v_{z} \approx 0$ at the X-point (by Mach probe measurement), the effective resistivity $\eta_{X}$ is calculated based on the following equations:

$$
\eta_{X} j_{t}=E_{t}+(\vec{v} \times \vec{B})_{t} \approx \frac{1}{2 \pi r_{x}} \frac{\Psi_{c o m}}{d t},
$$

where $E_{t}, j_{t}, \vec{B}, \vec{v}, \Psi_{\text {com }}$, and $r_{x}$ are toroidal electric field and toroidal current density at the X-point, magnetic field, plasma velocity, reconnected poloidal flux, and radius of the "X-point" line, respectively (Ono et al., 1993). Though the "effective" resistivity with particle or two fluid effect is not the real resistivity, it is still a useful indicator to interpret macroscopic reconnection physics based on the MHD approximation. In Fig. 6(a), the current sheet width $2 \delta$ is larger because of the weak external compressing force of $I_{a c c} \approx 5$ $\mathrm{kA}$. During the slow and weak compression process, $\eta_{X}$ is observed to be constant $(\approx 0.3 \mathrm{~m} \Omega \mathrm{m})$. However, $\eta_{X}$ increases slightly when $2 \delta$ becomes as short as $2 \rho_{i}$. In Fig. 6(b), the larger $I_{a c c} \approx 10 \mathrm{kA}$ provides the compression force large than that in (a) under the same $B_{X}$ condition. It is noted that $\eta_{X}$ is initially as small as (a) but that it increases significantly right after $2 \delta$ is compressed shorter than $2 \rho_{i} \approx 5 \mathrm{~cm}$ $(t>27.5 \mu \mathrm{sec})$. After $t=27.5 \mu \mathrm{sec}, 2 \delta$ is also observed to decrease faster than before.

In Fig. 6(c), the larger $B_{X} \approx 2.7 B_{\| 0}$ sets $2 \rho_{i}$ shorter than that in Fig. $6(\mathrm{~b})$. It is observed that $2 \delta$ is always longer than the small $2 \rho_{i}$ and that $\eta_{X}$ stays as small as $0.2-0.3 \mathrm{~m} \Omega \mathrm{m}$. No change in $2 \delta$ decaying speed occurs, even if $2 \delta$ is compressed shorter than $5 \mathrm{~cm}$.

Figures 7(a) and (b) show more clearly the relationship between anomalous increase in $\eta_{X}$ and $2 \delta$-compression shorter than $2 \rho_{i}$. In Fig. 7(a), fifteen time-evolutions of $\eta_{X}$ under four different $I_{a c c}$ are superposed on the same plot, in terms of $\delta$ normalized by $\rho_{i}$. All cases have equal $B_{X} / B_{\| 0}=1$. 

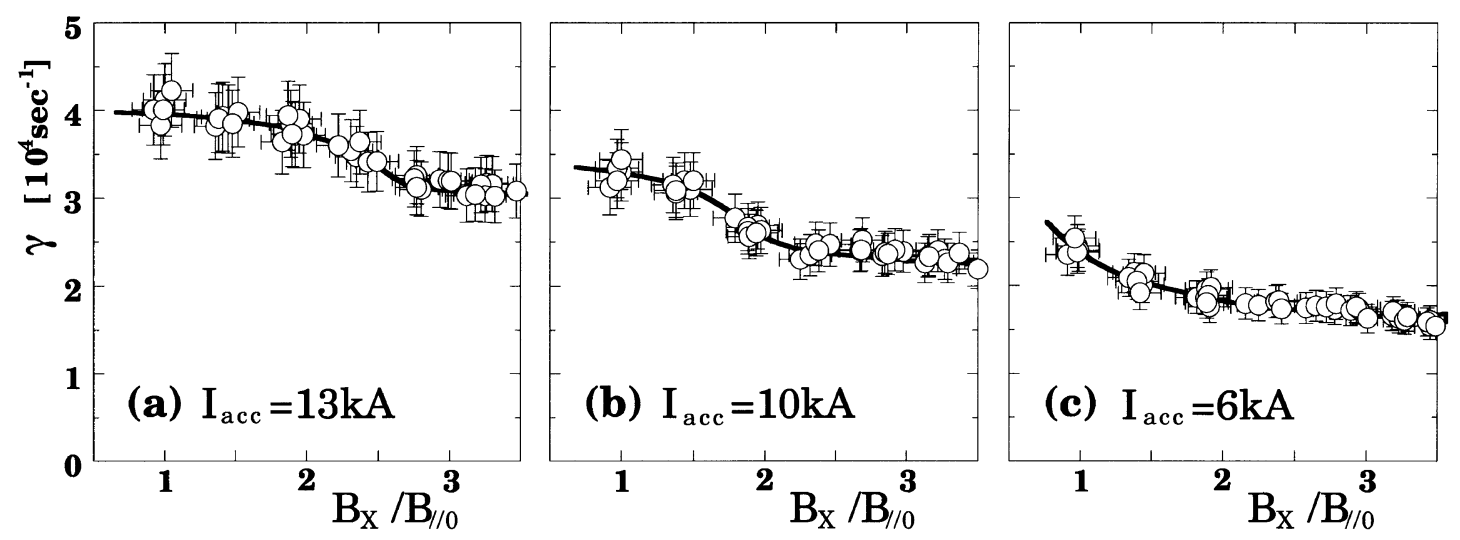

Fig. 4. Averaged reconnection rates $\gamma$ of two merging toroids as a function of $B_{X} / B_{\| 0}$ for three different acceleration-coil currents: (a) $I_{a c c}=13 \mathrm{kA}$, (b) $10 \mathrm{kA}$, and (c) $6 \mathrm{kA}$.
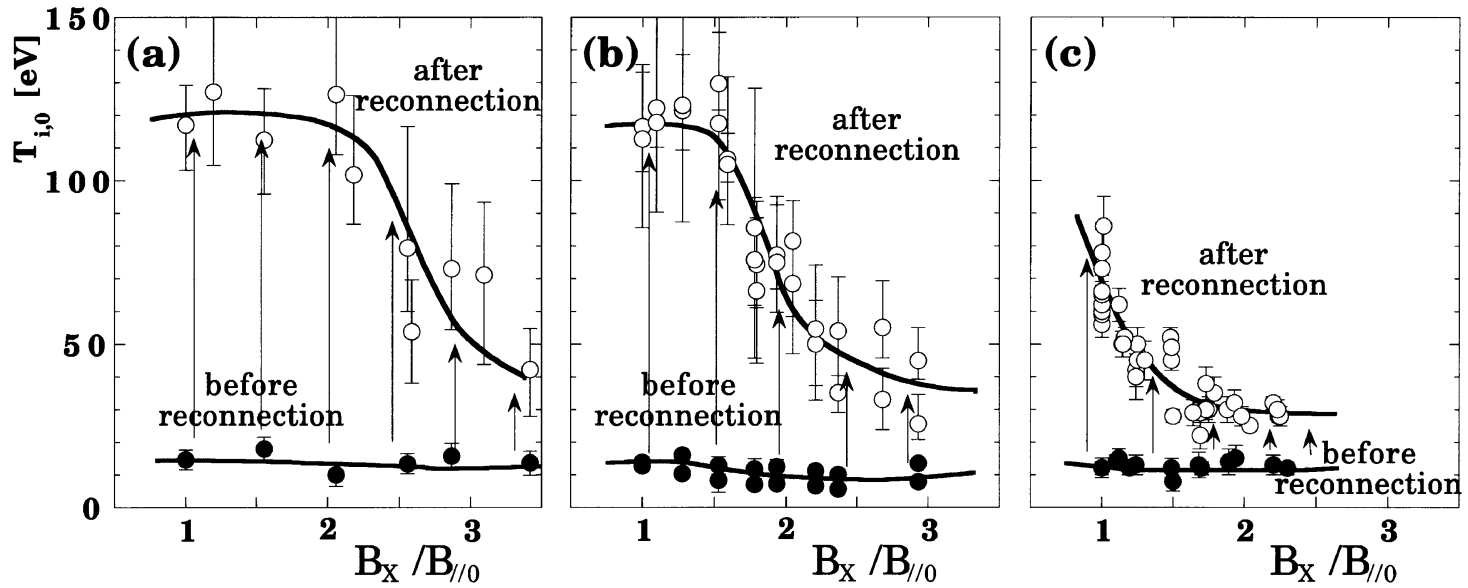

Fig. 5. Peak ion temperatures $T_{i 0}$ before and after the magnetic reconnection as a function of $B_{X} / B_{\| 0}$ for three different acceleration-coil currents: (a) $I_{a c c}=13 \mathrm{kA}$, (b) $10 \mathrm{kA}$, and (c) $6 \mathrm{kA}$.

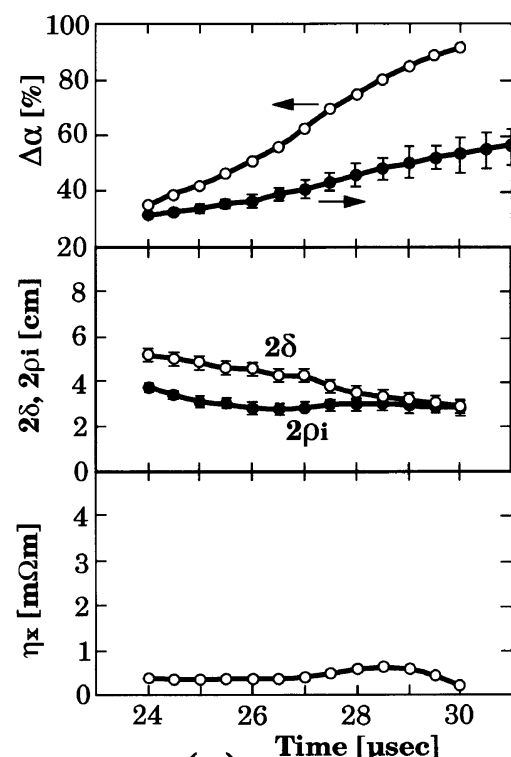

(a)

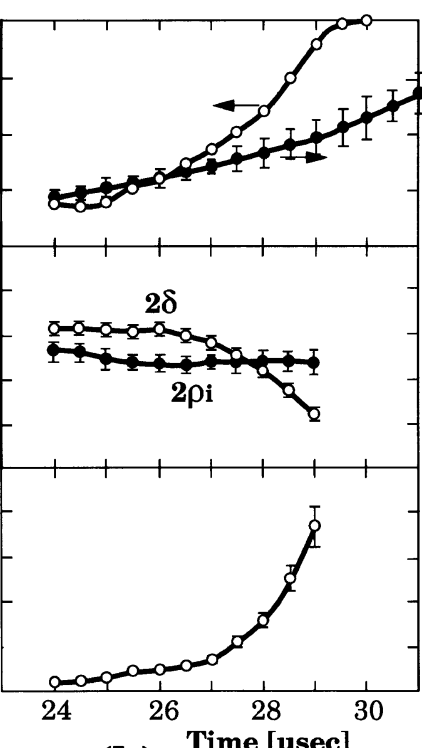

(b)

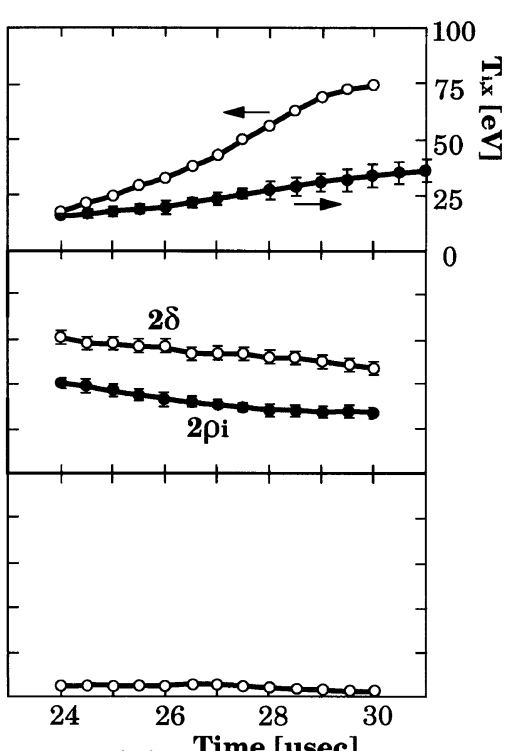

(c)

Fig. 6. Time evolution of common flux ratio $\alpha$, ion temperature $T_{i, X}$ at the X-point, current-sheet width $2 \delta$ and ion-gyroradius $\times 2$ (diameter) $2 \rho_{i}$, and effective resistivity $\eta_{X}$ for three different reconnections: (a) $B_{X} \approx 1.0 B_{\| 0}, I_{a c c} \approx 5 \mathrm{kA}$, (b) $B_{X} \approx 1.0 B_{\| 0}, I_{a c c} \approx 10 \mathrm{kA}$, and $(\mathrm{c}) B_{X} \approx 2.7 B_{\| 0}$, $I_{a c c} \approx 10 \mathrm{kA}$. 
(a)

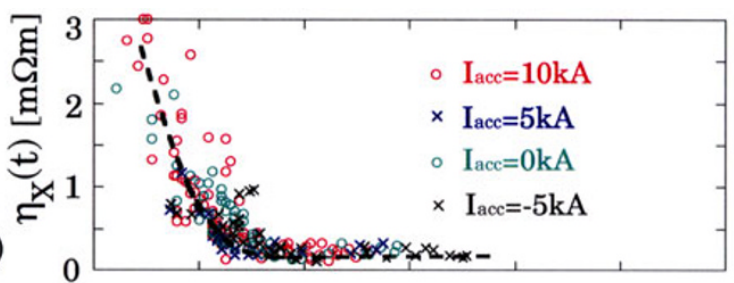

(b)

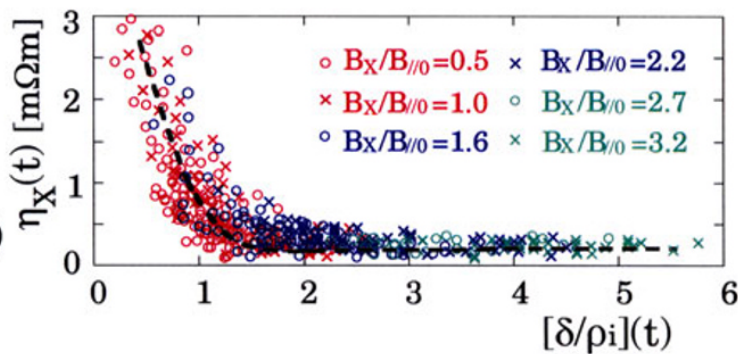

Fig. 7. Time evolution of effective current sheet resistivities $\eta_{X}$ as a function of $\delta$ normalized by $\rho_{i}$ when four different acceleration-coil currents $I_{a c c}$ (a) and six different $B_{X}$ fields (b) are applied to the merging toroids.

All curves agree in that $\eta_{X}$ stays as small as the Spitzer resistivity $\approx 0.2 \mathrm{~m} \Omega \mathrm{m}$ calculated from the measured electron temperature $\approx 5 \mathrm{eV}$, as long as $2 \delta$ is longer than $2 \rho_{i}$. However, $\eta_{X}$ increases significantly, once a large external force compresses $2 \delta$ shorter than $2 \rho_{i}$. The maximum $\eta_{X} \approx 3-3.5$ $\mathrm{m} \Omega \mathrm{m}$ at $\delta / \rho_{i} \approx 0.5$ is almost 15 times larger than the Spitzer resistivity, leading to the fast reconnection observed in the large $I_{a c c}$ regime. In Fig. 7(b), several evolutions of $\eta_{X}$ under six different $B_{X}$ field amplitudes are superposed to confirm the mentioned relationship. All cases have equal $I_{a c c}=10$ $\mathrm{kA}$. When $B_{X} / B_{\| 0}$ is varied, $\eta_{X}$ stays constant $(\approx 0.2 \mathrm{~m} \Omega \mathrm{m})$ when $2 \delta>2 \rho_{i}$ and becomes markedly large when $2 \delta<2 \rho_{i}$. Both curves in Figs. 7(a) and (b) agree well with each other, indicating that the anomalous increase in $\eta_{X}$ is correlated to $2 \delta$ being smaller than $2 \rho_{i}$.

\section{Discussion}

Both scan experiments of external-compression-force and $B_{X}$, consistently revealed the dependence of the effective sheet-current resistivity on $\delta$ normalized by $\rho_{i}$. Especially, it is important that $\eta_{X}$ in Fig. 6(a) is initially as small as (a) and (c) but that it increases significantly $(\partial B / \partial t \neq 0)$ right after $2 \delta$ is compressed shorter than $2 \rho_{i}$. This fact clearly indicates that possibly some particle effect causes the onset of anomalous current-sheet dissipation when $2 \delta$ becomes shorter than the threshold value: $2 \rho_{i}$. Initially, the thermal pressure of the current-sheet is considered to sustain the external compressing force of the magnetic field, maintaining the current sheet width. However, once the sheet width becomes shorter than the ion-gyroradius, ions no longer sustain the external force as a fluid, but start meandering inside and outside of the current-sheet unlike magnetized electrons. Horiuch and Sato studied the further sheet-compression processes with meandering ions using their macroparticle simulation. They observed that the reconnection speed increased significantly when the sheet width was compressed shorter than the iongyroradius (Horiuchi and Sato, 1997). Their results agree qualitatively with our observation mentioned above. They reported that the main cause for the anomalous sheet dissipation was the combination of lower hybrid drift instability and drift kink instability. The ion-gyroradius $\rho_{i}$ tends to be close to $c / \Omega_{p i}$ in the low- $B_{X}$ reconnection. We measured electron density $n_{e}$ of the current sheet with varied $B_{X}$ using electrostatic probes and observed that the effective resistivity is correlated with $\delta / \rho_{i}$, not with $\delta /\left(c / \Omega_{p i}\right)$ (Inomoto et al., 2000). The ion-gyroradius $\rho_{i}$ increases with increasing ion temperature and with decreasing magnetic field. This is the most probable reason why the reconnection speed increases with increasing external force and with decreasing $B_{X}$ in Figs. 4(a), (b), and (c). This result also suggests that change in the current sheet structure $(\partial B / \partial t \neq 0)$ is sometimes essential to the fast reconnection, in sharp contrast with slow and steady reconnection (Ji et al., 1998). In agreement with our observation, several MHD and particle simulations also reported that drastic changes in the sheet-structure and reconnection speed were triggered by increase in the effective resistivity (Ugai, 1992, 1994; Tajima et al., 1989).

The ion-heating-suppression effect of $B_{X}$ agrees with the general tendency that the $T_{i} / T_{e}$ ratio is roughly equal to unity in ohmic tokamak plasmas with $B_{X} \gg B_{\|}$, and much larger than unity in RFP and spheromak plasmas globally with $B_{X} \leq B_{\|}$. In small $B_{X}$ reconnection, the ion acceleration effect of magnetic field lines is considered to heat bulk ions selectively through the large ion viscosity effect. The unmagnetized ions are easily mixed together and are thermalized after the ion-acceleration phenomenon of reconnection. Since the ion-gyroradius is much larger than the electrongyroradius, this anomalous heating is considered to occur only for ions not for electrons. The merging process also compresses the effective minor radius of merging toroids by factor: $C \approx 1.3-1.4$, suggesting that the compression effect also contribute to the increase in $T_{i}$. However, its effect on $T_{i}$ increment $\approx C^{4 / 3} \approx 1.4-1.6$ is much smaller than the large (factor 10) difference in $T_{i}$ before and after the reconnection as shown in Fig. 5(a). On the other hand, the application of $B_{X}$ decreases the ion-gyroradius and magnetizes ions like electrons, preventing the ion-thermalization process. A decreases in ion-gyroradius and plasma outflow speed (reconnection speed) provide a possible explanation for the ion-heating-suppression effect of $B_{X}$, because the viscosity coefficient becomes smaller with increasing $B_{X}$ around the X-point. It is also noted that both the $T_{i}$ increments and reconnection rates become markedly large when $B_{X} / B_{\| 0}$ is smaller than the threshold values: 2.5 in Figs. 5(a), 1.8 in (b), and 1 in (c). In each case, the compressed $2 \delta$ is observed to reach $2 \rho_{i}$ when $B_{X} / B_{\| 0}$ is below each threshold value, indicating the close relationship between anomalous dissipation of the current sheet and the selective ion heating.

\section{Summary}

In summary, a series of experiments has elucidated the anomalous dissipation and heating effects of magnetic reconnection caused by current sheet compression. The sheetcurrent was observed to dissipate anomalously when the current sheet is compressed shorter than the ion-gyroradius around the X-point. Because of this effect, the reconnection speed and resistivity of the current-sheet increase with decreasing $B_{X}$ and with increasing external compressing force. 
This $B_{X}$ effect is consistent with the high magnetic fluctuations and $T_{i} \gg T_{e}$ characteristics of the RFPs whose reconnections have small $B_{X} \leq B_{\|}$globally. The application of $B_{X} \gg B_{\|}$suppresses this anomalous increase in sheet-current resistivity and ion temperature. This feature is consistent with low magnetic fluctuation and $T_{i} \approx T_{e}$ characteristics of tokamaks whose reconnecion has large toroidal magnetic field $B_{X} \gg B_{\|}$.

Acknowledgments. We would like to thank the TS-3 group: M. Katsurai, T. Okazaki, M. Ohi, S. Takeuchi, and A. Morita for experimental support and M. Yamada, R. Kulsrud, and H. Ji at Princeton Plasma Physics Laboratory for theoretical collaboration and useful discussions.

\section{References}

Balandin, A. et al., Design study of 3-D tomography diagnostics for spherical tokamaks, Eur. Phys. J., D14, 97-103, 2001.

Biskamp, D., Magnetic reconnection via current sheets, Phys. Fluids, 29, 1520-1531, 1986.

Gekelman, W. and H. Pfister, Experimental observations of the tearing of an electron current sheet, Phys. Fluids, 31, 2017-2025, 1988.

Horiuchi, R. and T. Sato, Particle simulation study of collisionless driven reconnection in a sheared magnetic field, Phys. Plasmas, 4, 277-289, 1997.

Inomoto, M. et al., Mechanism of fast reconnection in TS-3 plasma merging experiment, in Proc. MR2000, p. 23, Univ. Tokyo, Japan, Mar., 2000.

Ji, H. et al., Experimental test of the Sweet-Parker model of magnetic reconnection, Phys. Rev. Lett., 80, 3256-3259, 1998.

Leboeuf, J. N., T. Tajima, and J. M. Dawson, Dynamic magnetic X-point, Phys. Fluids, 25, 784-798, 1982.

Morita, A. et al., Tomotraphic reconstruction algorithm for an annular cross section of toroidal plasmas by use of modified Fourier-Bessel expansions, Trans. Inst. Electr. Eng. Japan, 115, 37-43, 1995.

Murata, Y. et al., Bulletin of American Physical Society 45, 7, YP1 53, 2000.

Ono, Y., A. Morita, M. Katsurai et al., Experimental investigation of threedimentional magnetic reconnection by use of two colliding spheromaks,
Phys. Fluids, B5, 3691-3701, 1993.

Ono, Y. et al., Ion acceleration and direct ion heating in three-component magnetic reconnection, Phys. Rev. Lett., 86, 3328-3331, 1996.

Parker, E. N., Mechanism for merging magnetic fields in conducting fluids, J. Geophys. Res., 62, 509-520, 1957.

Saito, Y. et al., Slow-mode shocks in magnetotail, J. Geophys. Res., 100, 23567-23581, 1995.

Sato, T. and T. Hayashi, Exterally driven reconnection and a powerful magnetic energy converter, Phys. Fluids, 22, 1189-1202, 1979.

Sato, T., T. Hayashi, K. Watanabe et al., Role of compressibility on driven magnetic reconnection, Phys. Fluids B, B4, 450-457, 1992.

Scime, E. et al., Ion heating and magnetohydrodynamic fluctuations in the reversed-field pinch, Phys. Fluids, 4, 4062-4071, 1992.

Shay, M. A. et al., Structure of the dissipation region during collisionless magnetic reconnection, J. Geophys. Res., 103, 9165-9176, 1998.

Shimizu, T., S. Tsuneta, and A. C. Acton, Morphology of active region transient brightenings with the $\mathrm{YOHKOH}$ soft X-ray telescope, Astrophys. J., 422, 906-911, 1994.

Stenzel, R. et al., Magnetic field line reconnection experiments 4. resistivity, heating and energy flow, J. Geophys. Res., 87, 111-117, 1982.

Sweet, P. A., The neutral point theory of solar flares, in Electromagnetic Phenomena in Cosmical Physics, edited by B. Larnert, p. 123, Cambridge, New York, 1953.

Tajima, T. and J.-I. Sakai, Explosive coalescence of magnetic islands and acceleration of changed particles, Sov. J. Plasma Phys., 15, 519-525, 1989.

Treumann, R. A. and W. Baumjohann, Advanced Space Plasma Physics, Imperial College Press, 1997.

Tsuneta, S., Interacting active regions in the solar corona, Astrophys. J., 456, L63-L65, 1996.

Ugai, M., Computer studies on development of the fast reconnection mechanism for different resistivity model, Phys. Fluids, B4, 2953-2963, 1992.

Ugai, M., Computer studies on fast reconnection mechanism under an externally driven boundary condition, Phys. Plasmas, 1, 2853-2863, 1994.

Yamada, M. et al., Identification of Y-shaped and O-shaped diffusion regions during magnetic reconnection in a laboratory plasma, Phys. Rev. Lett., 78, 3117-3120, 1997.

Y. Ono (e-mail: ono@katsurai.t.u-tokyo.ac.jp), M. Inomoto, Y. Ueda, T. Matsuyama, and Y. Murata 